\title{
Revealing the Decrease of Renal Cortical Perfusion in Primary Glomerular Disease and Renal Aging by Arterial Spin Labeling
}

\author{
Chenxia Li ${ }^{1,2}$, Xiang $\mathrm{Li}^{1}$, Haitian Liu ${ }^{3}$, Rong Wang ${ }^{1}$, Gang Niu ${ }^{1}$, Jian Yang ${ }^{1,2}$ and Yuelang Zhang ${ }^{1, *}$ \\ ${ }^{1}$ Department of Radiology, The First Affiliated Hospital of Xi'an Jiaotong University, Xi'an, PR China \\ ${ }^{2}$ Department of Biomedical Engineering, The Key Laboratory of Biomedical Information Engineering of the Ministry of Education, School of Life Science and Technology, \\ Xi'an Jiaotong University, Xi'an, PR China \\ ${ }^{3}$ Department of Radiology, The Third Hospital of Hebei Medical University, Shijiazhuang, PR China \\ "Corresponding author: Department of Radiology, The First Affiliated Hospital of Xi'an Jiaotong University, No.277 Yanta West Rd., Xi'an 710061, PR China. Tel: +86-18991232590, \\ Fax:+86-02985225009, Email: zhyl_003@163.com
}

Received 2019 July 08; Revised 2020 December 23; Accepted 2019 December 28.

\begin{abstract}
Objectives: To reveal the change of renal cortical blood perfusion in primary glomerular disease (PGD) and renal aging by flowsensitive alternating inversion recovery (FAIR) arterial spin labeling (ASL) technique.

Patients and Methods: Renal cortical perfusion was estimated in 24 PGD patients and 30 healthy volunteers using FAIR-ASL in this case-control study. MRI examination was repeated after one week in six randomly selected healthy volunteers. In PGD patients, we regard estimated glomerular filtration rate (eGFR) $\geq 60 \mathrm{~mL} / \mathrm{min} / 1.73 \mathrm{~m}^{2}$ as mild PGD and eGFR $<60 \mathrm{~mL} / \mathrm{min} / 1.73 \mathrm{~m}{ }^{2}$ as moderatelate PGD. The correlations between ASL perfusion values and eGFR in healthy volunteers, mild and moderate-late PGD patients were assessed.

Results: In healthy volunteers, the perfusion values between the twice ASL scanning with one week-interval showed an excellent agreement by Bland-Altman analysis. The mean perfusion value of the bilateral kidneys decreased with age increase ( 25 - 72 years) $(\mathrm{r}=-0.496, \mathrm{P}=0.012)$. There were significant differences in ASL perfusion values among healthy volunteers, mild and moderate-late PGD patients $(\mathrm{P}<0.0001)$. Perfusion values correlated strongly with eGFR in all PGD patients $(\mathrm{r}=0.868, \mathrm{P}<0.0001)$, but not in healthy volunteers $(r=0.156, P=0.405)$. The correlation coefficient of perfusion values with eGFR in mild and moderate-late PGD patients were $0.749(\mathrm{P}=0.008)$ and $0.809(\mathrm{P}=0.010)$, respectively.

Conclusion: FAIR-ASL technique represents a feasible approach to monitor the renal perfusion variations in PGD patients. It is a noninvasive technique to discover the early abnormality of renal function, and assess the degree of renal aging.
\end{abstract}

Keywords: Chronic Kidney Disease, Magnetic Resonance Imaging, Arterial Spin Labeling, Glomerular Filtration Rate, Renal Function

\section{Background}

Chronic kidney disease (CKD) is an increasing public health issue, and prevalence is estimated to be $8 \%$ $16 \%$ worldwide (1). An epidemiological study indicates the number of patients with CKD in Chinese adults is estimated to be about 119.5 million, in which mild CKD occupies more than $99 \%$ of the total number (2). Symptoms of mild CKD are not obvious and often overlooked, which is the leading cause of end stage renal disease and death.

Hemodynamic changes have been described in early stages of CKD (3). Serum creatinine, glomerular filtration rate (GFR), and urine albumin-creatinine ratio are clinical tools used for evaluation of renal function, while they are not sensitive enough to detect early functional disturbances and monitor hemodynamic changes (4). The arterial spin labeling (ASL) perfusion MRI, in which inflowing blood is labeled magnetically, can be used in spatial quantification of renal blood flow (RBF) to assess renal function (5).

A flow-sensitive alternating inversion recovery (FAIR) sequence is a promising technique for grading renal artery stenosis in native kidneys, assessing acute kidney injury and function of transplanted kidneys (6). At present, only a few studies have investigated ASL measurements in patients with CKD. It has been detected that the distribution of cortical perfusion values using ASL is associated with renal dysfunction, cortical and renal perfusion could distinguish healthy volunteers from CKD patients, and it can evaluate renal blood flow in stage III CKD (7). However, there is no study investigating its value in identifying different stage of $\mathrm{CKD}$, especially in primary glomerular disease (PGD) which is the most common type of CKD. 


\section{Objectives}

In this study, we aim to investigate the feasibility of FAIR-ASL technique to monitor the variation of renal perfusion in PGD and explore its value for identifying mild PDG from healthy and moderate-late PGD.

\section{Patients and Methods}

\subsection{Ethics Statement}

This case-control study was approved by the Ethics Committee of our hospital. Informed consent was obtained from all individual subjects included in the study.

\subsection{Subjects}

All subjects were consecutively recruited from December 2015 to June 2016 in the west of China. Healthy volunteers were recruited from the society through various channels, including graduate students, medical workers and healthy volunteers outside the hospital. They were included based on the following criteria: (1) adults ( $\geq 18$ years old); (2) no hypertension and diabetes or other systemic diseases; (3) no clinical signs of kidney disease, including low back pain, hematuria, edema and urinary irritation; (4) no contraindications to 3.0 T MRI and good cooperation during the examination; and (5) no family history of kidney disease. Participants were excluded based on the following criteria: (1) poor MRI image quality; and (2) renal artery stenosis.

Inclusion criteria for PGD patients were as follows: (1) adults ( $\geq 18$ years old); (2) meeting international standard of clinical CKD diagnosis; (3) no contraindications to 3.0 T MRI and good cooperation during the examination; (4) not taking medicine influencing creatinine measurements (such as cimetidine or cephalosporin); (5) not receiving any form of renal replacement therapy. Exclusion criteria were as follows: (1) renal angiomyolipoma; (2) poor MRI image quality; (3) skeletal muscle atrophy, pleural effusion or ascites, malnutrition, amputation, heart failure, ketoacidosis and other severe systemic diseases; (4) glomerular disease was not confirmed by needle biopsy.

\subsection{MRI Protocols}

All MRI scanning was performed on a 3.0T scanner (Signa HDxt, General Electric Medical System, USA) using an eight-channel phased array coil. Prior to scanning, all subjects fasted for at least 6 hours and were taught to perform end-expiratory breath holds. Conventional T1 weighted imaging (T1WI), T2 weighted imaging (T2WI) and FAIR-ASL were performed. T1WI was performed using a fast spoiled gradient echo (FSPGR) sequence with the following parameters: repetition time $(\mathrm{TR})=4 \mathrm{~ms}$, echo time $(\mathrm{TE})=1.2$ $\mathrm{ms} / 2.4 \mathrm{~ms}$, slice thickness $/ \mathrm{spacing}=8 \mathrm{~mm} / 1 \mathrm{~mm}$, matrix size $=192 \times 256$, flip angle $=80^{\circ}$, field-of-view $($ FOV $)=38-42$ $\mathrm{cm}$. T2WI was performed for anatomical evaluation using a single shot fast spin echo (SSFSE) technique with the following parameters: $\mathrm{TR}=10,000 \mathrm{~ms}, \mathrm{TE}=86 \mathrm{~ms}$, slice thickness $/$ spacing $=5 \mathrm{~mm} / 2 \mathrm{~mm}$, matrix size $=256 \times 180$, flip angle $=90^{\circ}$ and $\mathrm{FOV}=38-42 \mathrm{~cm}$.

FAIR sequence with a standard echo-planar imaging (EPI) acquisition was used, combined with array spatial sensitivity encoding technique (ASSET) under breathholding conditions to minimize motion artifacts. Four slices were prescribed using renal hilum as center. Two pairs of control/label phases were acquired during endexpiratory breath hold, which produced totally 16 images, including eight label images with section selective inversion and eight control images with non-selective global inversion. Total scan time is $16 \mathrm{~s}$. Acquisition parameters were as follows: $\mathrm{TR}=4400 \mathrm{~ms} ; \mathrm{TE}=11.9-12.3 \mathrm{~ms}$; FOV $=38$ - $42 \mathrm{~cm}$; matrix size $=128 \times 128$; slice thickness $/$ spacing $=5$ $\mathrm{mm} / 2 \mathrm{~mm}$, slices $=4 ;$ ASSET factor $=2$, flip angle $=90^{\circ} ;$ labeling delay time $(\mathrm{LDT})=1,200 \mathrm{~ms}$ and marker slice thickness $=53 \mathrm{~mm}$. The first pair of control/label phase was discarded during perfusion calculation because it did not reach steady state. Six of the healthy volunteers were selected randomly for repeated MRI examination after one week to investigate the repeatability of FAIR-ASL.

\subsection{MR Imaging Process}

The original MRI data were transferred to a postprocessing GE AW 4.4 workstation. A quantitative model for perfusion rate estimation based on extended block equations (8) was used to obtain a difference image $(\Delta M)$, which indicated different longitudinal magnetization between label and control images. A perfusion map was generated by using $\Delta \mathrm{M}$ and $\mathrm{M}_{0}$ from each pixel. Perfusion rate was calculated according to the following formula:

$f=\frac{\lambda}{2 \alpha \cdot L D T} \cdot \frac{\Delta \mathrm{M}}{\mathrm{M}_{0}} \cdot \exp \left(\frac{L D T}{\mathrm{~T}_{1}}\right)$

Where $\mathrm{f}$ is perfusion rate $(\mathrm{mL} / 100 \mathrm{~g} / \mathrm{min}) ; \lambda$ is bloodtissue water partition coefficient (nearly constant at 80 $\mathrm{mL} / 100 \mathrm{~g}$ ), $\alpha=1.0$ (inversion efficiency), and $\mathrm{M}_{0}$ is tissue equilibrium magnetization per unit of tissue, $\mathrm{LDT}=1,200$ $\mathrm{ms}$, and $\mathrm{T}_{1}=1142 \mathrm{~ms}$ for cortex.

In this study, only perfusion values in renal cortex were measured because cortical perfusion accounts for more than $90 \%$ of renal perfusion. Regions of interest (ROI) was selected as follows: three oval ROIs with an area of 50-100 $\mathrm{mm}^{2}$ were positioned at anterior, middle and posterior renal cortex on axial image. The ROIs were copied and applied as similarly as possible to bilateral kidney. No deformations or artifacts were found at the measuring level. The kidney edge, blood vessel, renal pelvis, renal calyces 
and renal sinus fat were also avoided. To avoid the influence of feeding vessels, pixels with perfusion values of over $450 \mathrm{~mL} / 100 \mathrm{~g} / \mathrm{min}$ were not included in analysis (9). All measurements were performed independently by two experienced radiologists (17 and 8 years of experience in MR imaging) blinded to volunteer and patient information. All measurements were completed within two weeks after the MRI examination. The average value was calculated according to all measurements of the single and bilateral kidney.

\subsection{Estimated Glomerular Filtration Rate (eGFR) Measurement}

The K/DOQI clinical practice guidelines recommend eGFR as the evaluation index of renal function, which is based on serum creatinine levels and is the most widely used clinical method for assessment of general renal function (10). Ten milliliter venous blood samples were collected from all healthy volunteers and PGD patients for serum creatinine measurement within three days before or after MRI examination. The eGFR was calculated using serum creatinine concentration and demographics according to the modified diet in renal disease (MDRD) formula for Chinese individuals (11):

$$
\begin{aligned}
& e G F R=186 \times S c r^{-1.154} \times a g e^{-0.203} \\
& \times 0.724 \text { (iffemale }) \times 1.233(\text { ifChinese }) \\
& \text { Scr }=\text { serum creatinine }(\mathrm{mg} / \mathrm{dL})
\end{aligned}
$$

All patients with PGD were staged by the K/DOQI clinical practice guidelines and divided into two groups according to eGFR value: high eGFR group (HGFR) (stage I - II with eGFR $\geq 60 \mathrm{~mL} / \mathrm{min} / 1.73 \mathrm{~m}^{2}$, mild PGD) and low eGFR group (LGFR) (stage III - V with eGFR $<60 \mathrm{~mL} / \mathrm{min} / 1.73 \mathrm{~m}^{2}$, moderate-late PGD).

\subsection{Statistical Analysis}

Continuous variables (e.g. eGFR) were represented as mean \pm standard deviation(SD). The number of binary categorical variables (e.g. gender) was reported and its percentage presented in parenthesis. Bland-Altman plot was constructed by MedCalc 11.3 to evaluate the repeatability of MRI examinations before and after one-week interval and the inter-observer consistency. The difference in perfusion values of left and right kidney was compared by paired $t$ test. Binary categorical variables (e.g. gender) of patient and healthy volunteers were compared by $\chi^{2}$ test. The differences of continuous variables such as age, eGFR and perfusion values in control, HGFR and LGFR groups were compared by analysis of variance (one-way ANOVA), and multiple comparisons between the groups were performed using Student-Newman-Keuls test. Spearman's correlation test was used to determine the relationship between renal cortical perfusion and age or eGFR. These statistical analyses were performed using SPSS 20.0.0 (IBM Corp. Released 2011. IBM SPSS Statistics for Windows, Version 20.0. Armonk, NY).

$\mathrm{P}<0.05$ was considered as statistical significance.

\section{Results}

\subsection{Clinical Information of Healthy Controls and PGD Patients}

The flow chart for screening of healthy controls and PGD patients is shown in Figure 1. Thirty healthy volunteers and 24 patients with PGD were enrolled. The histological results of 16 patients were obtained by renal biopsy at 4.82 \pm 2.93 days after MRI examination, and the other subjects were derived from renal biopsy 2 years ago. The comparison of clinical information of healthy volunteers and PGD is demonstrated in Table 1 . Significant differences in eGFR were observed among control, HGFR and LGFR groups ( $P$ $<0.05)$. There were no significant differences in age and gender of subjects among the three groups $(\mathrm{P}>0.05)$. Detailed clinical information and demographic data of PGD patients are shown in Table 2. Eleven patients in CKD stage I - II (HGFR group), and 13 patients in CKD stage III - V (LGFR group) have been mentioned. Representative ASL image of the ROI positioning is shown in Figure 2. Bland-Altman analysis showed excellent repeatability of perfusion values in renal cortex between two ASL scans and inter-observer consistency, with a random distribution of means plotted against differences observed (Figure 3).

\subsection{Renal Cortical Perfusion Analysis in Healthy Volunteers}

In healthy volunteers, there was no significant difference between mean cortical perfusion values in left $(299.50$ $\pm 45.44 \mathrm{~mL} / 100 \mathrm{~g} / \mathrm{min})(\mathrm{n}=30)$ and right $(302.64 \pm 49.94$ $\mathrm{mL} / 100 \mathrm{~g} / \mathrm{min})(\mathrm{n}=30)$ kidneys $(\mathrm{P}>0.05)$. The mean perfusion value of bilateral kidneys is $304.50 \pm 49.05 \mathrm{~mL} / 100$ $\mathrm{g} / \mathrm{min}$ which decreased with the increase in age $(\mathrm{r}=-0.496$, $\mathrm{P}=0.012$ ) (Figure 4). However, there was no correlation between ASL perfusion values and eGFR in healthy volunteers $(r=0.156, P=0.405)$.

\subsection{Renal Cortical Perfusion Analysis in PGD Patients}

Among 24 PGD patients, there was no significant difference in the mean perfusion value of left $(203.75 \pm 52.94$ $\mathrm{mL} / 100 \mathrm{~g} / \mathrm{min})(\mathrm{n}=24)$ and right $(207.16 \pm 53.48 \mathrm{~mL} / 100$ $\mathrm{g} / \mathrm{min})(\mathrm{n}=24)$ kidneys $(\mathrm{P}>0.05)$. The mean perfusion value was $247.86 \pm 34.90 \mathrm{~mL} / 100 \mathrm{~g} / \mathrm{min}$ in HGFR group and $172.82 \pm 42.08 \mathrm{~mL} / 100 \mathrm{~g} / \mathrm{min}$ in LGFR group. There were significant differences in renal cortical perfusion values among healthy control, HGFR and LGFR groups $(\mathrm{P}<0.05)$ (Figure 5). Furthermore, a significant correlation was identified between perfusion value and eGFR in PGD patients ( $r$ 


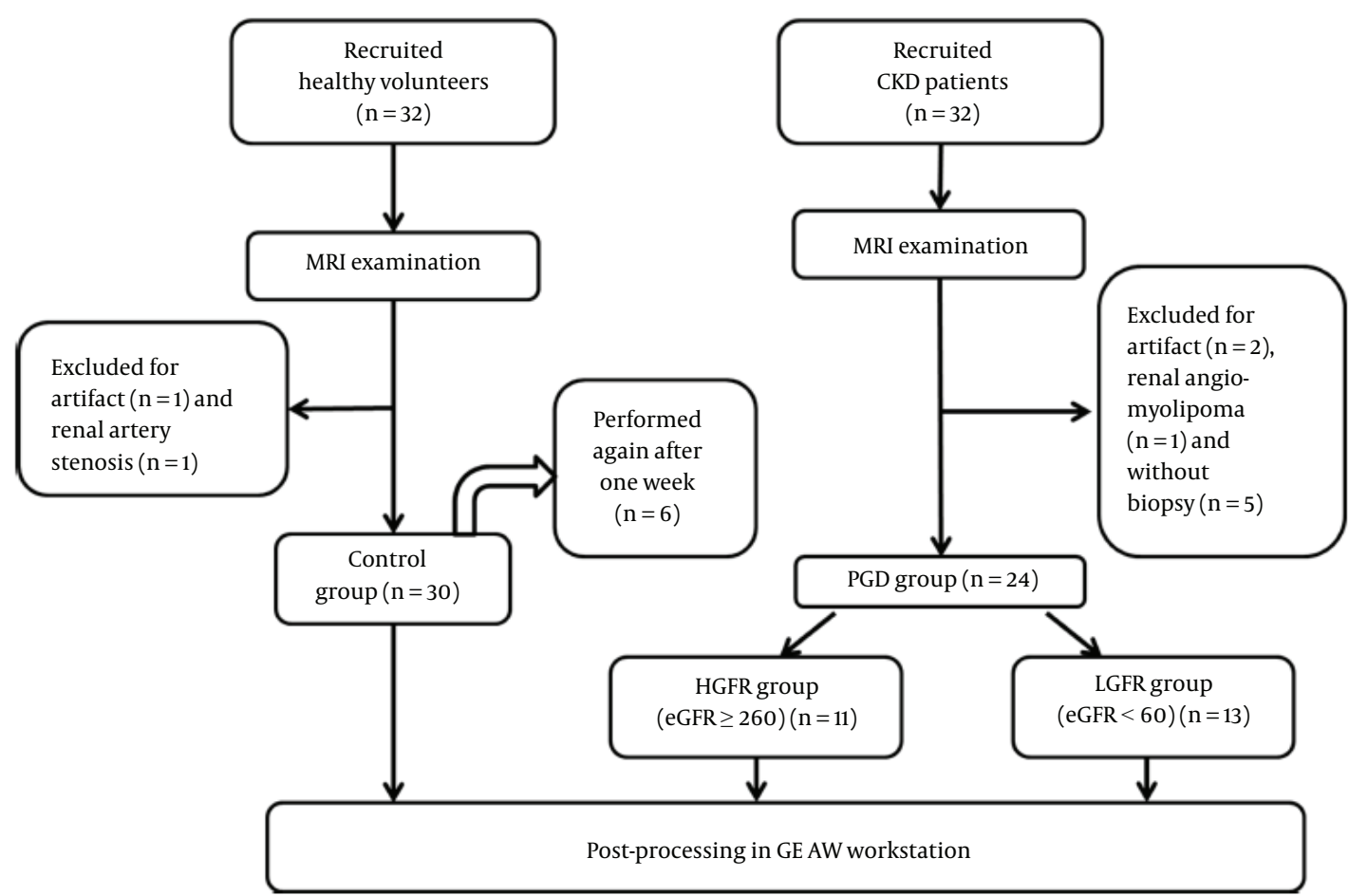

Figure 1. Flow chart for recruitment of healthy volunteers and PGD patients. (CKD, chronic kidney disease; PGD, primary glomerular disease; eGFR, estimated glomerular filtration rate; HGFR, high eGFR; LGFR, low eGFR)

Table 1. Comparison of Clinical Information of Healthy Volunteers and PGD Patients ${ }^{\mathrm{a}}$

\begin{tabular}{|c|c|c|c|c|}
\hline & \multirow{2}{*}{ Control } & \multicolumn{2}{|c|}{ PGD patients } & \multirow{2}{*}{ Pvalue } \\
\hline & & eGFR $\geq 60 \mathrm{~mL} / \mathrm{min} / 1.73 \mathrm{~m}^{2}$ & eGFR $<60 \mathrm{~mL} / \mathrm{min} / 1.73 \mathrm{~m}^{2}$ & \\
\hline Male & $17(56.67)$ & $8(72.73)$ & $9(69.23)$ & 0.28 \\
\hline Age, $y$ & $38.90 \pm 14.92$ & $44.51 \pm 15.34$ & $39.62 \pm 12.81$ & 0.40 \\
\hline eGFR, $\mathrm{mL} / \mathrm{min} / 1.73 \mathrm{~m}^{2}$ & $110.95 \pm 11.01$ & $106.20 \pm 26.23$ & $25.13 \pm 16.62$ & $<0.01^{\mathrm{b}}$ \\
\hline Serum creatinine, $\mu \mathrm{mol} / \mathrm{L}$ & $79.66 \pm 12.42$ & $85.95 \pm 14.61$ & $512.64 \pm 510.31$ & $0.01^{\mathrm{b}}$ \\
\hline Urea nitrogen, $\mathrm{mmol} / \mathrm{L}$ & I & $4.45 \pm 1.34$ & $17.53 \pm 9.91$ & $<0.01^{\mathrm{b}}$ \\
\hline Urea acid, $\mu \mathrm{mol} / \mathbf{L}$ & 1 & $497.12 \pm 156.14$ & $495.57 \pm 59.71$ & 0.98 \\
\hline Urea protein, g/d & 1 & $2.83 \pm 1.74$ & $3.32 \pm 1.93$ & 0.52 \\
\hline
\end{tabular}

Abbreviations: eGFR, estimated glomerular filtration rate; PGD, primary glomerular disease; SD, standard deviation

${ }^{a}$ Values are expressed as mean \pm SD or No. (\%)

${ }^{\mathrm{b}} \mathrm{P}<0.05$

$=0.868, \mathrm{P}<0.0001$ ) (Figure 6A). Compared with the control group, perfusion value was reduced by $18.6 \%$ in the HGFR group and by $43.2 \%$ in the LGFR group. Excellent correlation was also found if PGD patients were subgrouped according to the eGFR cut-off value of $60 \mathrm{~mL} / \mathrm{min} / 1.73 \mathrm{~m}^{2}$ (HGFR group: $r=0.749, \mathrm{P}=0.008$; LGFR group: $\mathrm{r}=0.809, \mathrm{P}$ $=0.010)$ (Figure 6B and C).

\section{Discussion}

In this study, FAIR-ASL technique was adopted under breath-holding condition to evaluate kidney perfusion in PGD patients. The perfusion value of kidneys decreased with the increase in age (25 - 72 years). The renal cortical perfusion values derived from FAIR-ASL displayed significant differences among healthy volunteers, mild and moderate-late PGD patients. Moreover, it correlated with eGFR in PGD patients obviously. The feasibility of FAIR-ASL 


\begin{tabular}{|c|c|c|c|c|c|c|}
\hline No. & Gender & Age, $y$ & eGFR, $\mathrm{mL} / \mathrm{min} / 1.73 \mathrm{~m}^{2}$ & Perfusion, $\mathrm{mL} / \mathrm{min} / 100 \mathrm{~g}$ & CKD stage & Histopathological classification \\
\hline $\mathbf{1}$ & Male & 63 & 104 & 244.13 & I & Membranous nephropathy I-II \\
\hline 2 & Male & 37 & 139 & 255.06 & I & Membranous nephropathy II \\
\hline 3 & Male & 38 & 126 & 302.03 & I & IgA nephropathy with focal hyperplasia \\
\hline 4 & Female & 59 & 96 & 216.27 & I & Membranous nephropathy II \\
\hline 5 & Male & 52 & 98 & 270.27 & I & IgA nephropathy \\
\hline 6 & Male & 25 & 144 & 307.94 & I & Atypical membranous nephropathy \\
\hline 7 & Male & 23 & 102 & 213.29 & I & IgA nephropathy \\
\hline 8 & Male & 43 & 136 & 261.04 & I & Membranous nephropathy II \\
\hline 9 & Female & 28 & 82.7 & 211.42 & II & Mesangial proliferative glomerulonephritis \\
\hline 10 & Female & 61 & 68 & 215.79 & II & Membranous nephropathy I-II \\
\hline $\mathbf{1 1}$ & Male & 60 & 73 & 229.27 & II & IgA nephropathy with mesangial proliferation \\
\hline 12 & Male & 49 & 50 & 204.2 & III & Membranous nephropathy III \\
\hline 13 & Male & 29 & 30 & 217.01 & III & IgA nephropathy with hyperplastic sclerosis \\
\hline 14 & Male & 47 & 46 & 209.79 & III & Mesangial proliferative glomerulonephritis \\
\hline 15 & Female & 48 & 43.5 & 186.25 & III & Focal hyperplastic sclerosing glomerulonephritis \\
\hline 16 & Male & 41 & 47 & 212.3 & III & IgA nephropathy \\
\hline 17 & Female & 48 & 28 & 220.79 & IV & IgA nephropathy with hyperplastic sclerosis \\
\hline 18 & Male & 62 & 20 & 190.54 & IV & Mesangial proliferative glomerulonephritis \\
\hline 19 & Male & 29 & 12 & 164.01 & $\mathrm{~V}$ & Membranous nephropathy III \\
\hline 20 & Male & 26 & 10 & 123.54 & V & Membranous nephropathy II \\
\hline 21 & Female & 41 & 11 & 118.33 & $\mathrm{~V}$ & IgA nephropathy with hyperplastic sclerosis \\
\hline 22 & Male & 28 & 13.7 & 141.8 & V & IgA nephropathy with hyperplastic sclerosis \\
\hline 23 & Male & 50 & 13 & 163.18 & $\mathrm{~V}$ & Focal segmental glomerular sclerosis \\
\hline 24 & Female & 17 & 2.4 & 94.94 & $\mathrm{~V}$ & $\begin{array}{c}\text { Membranous nephropathy III with glomerular sclerosis } \\
\text { glomerular sclerosis }\end{array}$ \\
\hline
\end{tabular}

Abbreviations: eGFR, estimated glomerular filtration rate; CKD, chronic kidney disease; PGD, primary glomerular disease

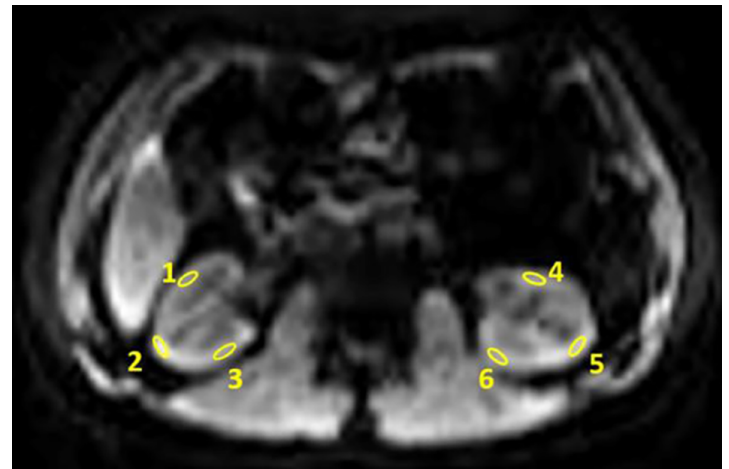

Figure 2. Representative image of the regions of interest (ROI) positioning in anatomical arterial spin labeling (ASL) image after selective inversion. Three oval ROIs with an area of $50-100 \mathrm{~mm}^{2}$ were positioned at the anterior, middle and posterior renal cortex on axial image, and the ROIs were copied and applied as similarly as possible to the bilateral kidney.

technique to monitor renal function in PGD patients was associated with the repeatability and accuracy of its perfusion measurements. Our study showed that the perfusion obtained from FAIR-ASL in healthy volunteers had ex- cellent repeatability, which was consistent with previous studies (12). It is known that motion artifact in ASL is an important technical issue related to renal perfusion quantification (13). Therefore, the end-expiratory breath-holding ASSET technique was adopted in this study to avoid the effect of motion and ensure the stability of FAIR-ASL. In the present study, axial positions were used which was different from most of previous studies. This was because there was no significant difference between the perfusion values of the coronal sections and the perfusion values of the axial sections in five healthy volunteers in our preliminary FAIRASL study, which is consistent with the results reported by Karger et al. (9). The mean cortical perfusion of healthy volunteers in the current study was $304.50 \pm 49.05 \mathrm{~mL} / 100$ $\mathrm{g} / \mathrm{min}$, which is in accordance with the findings in several previous studies (14). So the FAIR-ASL technique could reliably reflect renal perfusion quantification.

Our result showed that there was moderate correlation between perfusion values and age in healthy volunteers. This is because senile renal vascular changes occur with age, such as renal atherosclerosis, vascular dysautonomia, arteriole subendothelial hyalinosis and aglomerular circulation corresponding with age increase. All of these would 

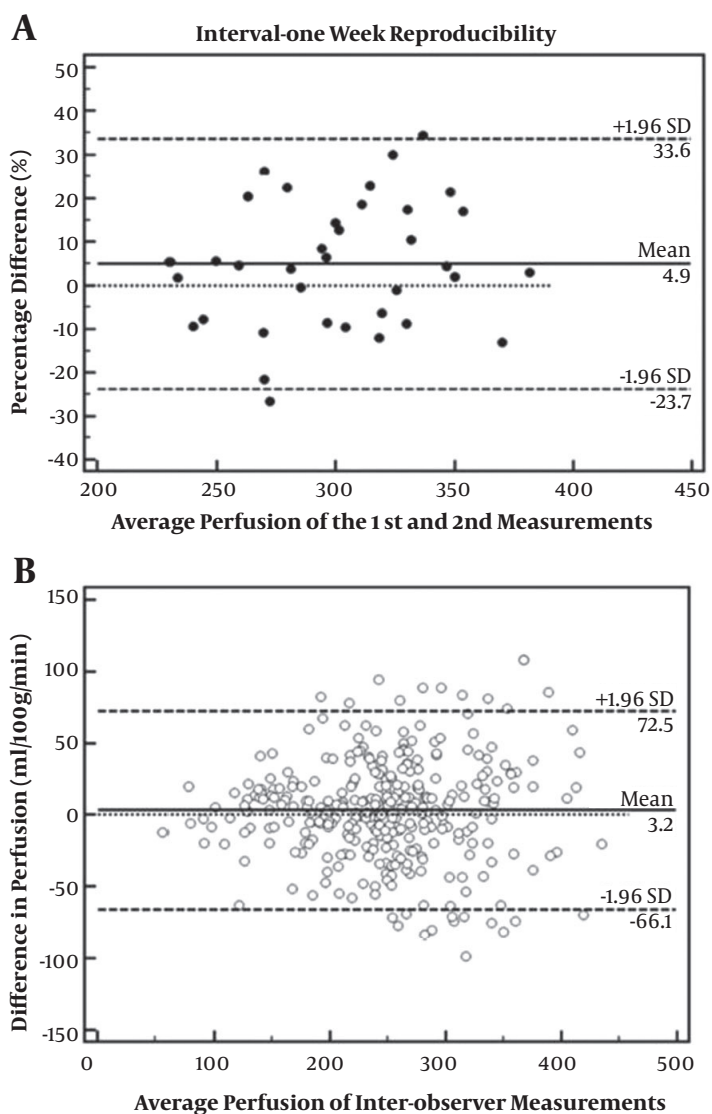

Figure 3. Bland-Altman plots of renal perfusion values from two arterial spin labeling (ASL) scans (A) and two observers measurements (B). The central dashed line is the line of equality (difference $=0$ ). The central solid line represents mean absolute difference, with $95 \%$ confidence interval of mean difference for two measurements (dashed lines around). Both 95\% confidence intervals and mean values from $A$ and B were close to zero.

result in the decrease of effective renal plasma flow and GFR. After the age of 30 years, the process of glomerular replacement by fibrous tissue corresponds to a decrease in the number of glomeruli, and GFR declines progressively to an approximate rate of $8 \mathrm{~mL} / \mathrm{min} / 1.73 \mathrm{~m}^{2}$ per decade (15). Thus, it could be suggested that the cortical perfusion value acquired by FAIR-ASL could provide a non-invasive and non-radioactive approach for assessing the degree of kidney aging, which might be useful for evaluating the donor renal function in renal transplantation.

Symptoms of mild CKD are not obvious and often overlooked, so diagnosis of mild PDG and appropriate intervention is helpful to improve the prognosis of patients. Our results revealed that FAIR-ASL might be a promising technique to discover the early hemodynamic changes of renal function and assist the diagnosis of mild PGD. It might be related to the alterations of renal physiology in progressive PGD. The glomerular injury results in oblitera-

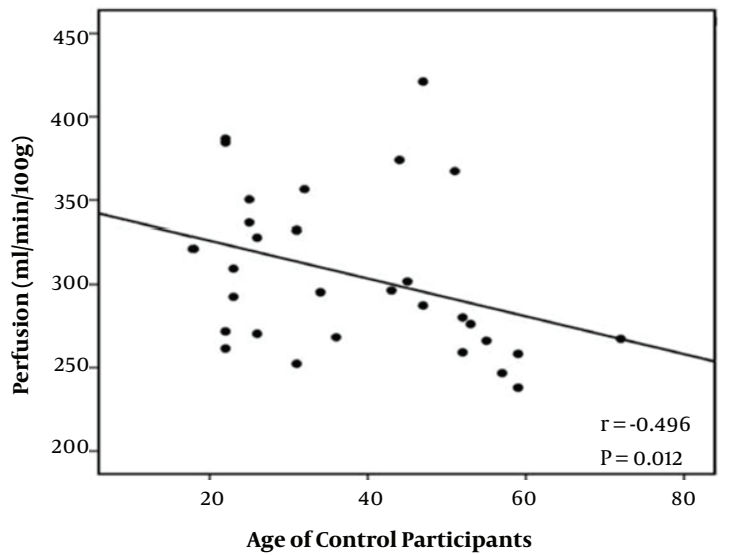

Figure 4. Spearman's correlation analysis of age with perfusion in healthy volunteers. The cortical perfusion values of healthy volunteers correlated negatively with the increase in age.

tion of the glomerular microvasculature and downstream peritubular capillaries, leading to renal tissue hypoperfusion. Rossi et al. (16) found that normal volunteers had significantly higher perfusion values than those of mildmoderate CKD patients. Our result further demonstrated that there are significant differences among health volunteers, mild (stage I - II) and moderate-late (stage III - V) PGD patients. Besides, perfusion values obtained by this study correlated with eGFR in all PGD patients and subgroup PGD patients markedly, which was consistent with several studies (7). Moreover, the correlation coefficients obtained by this study was higher than others. We speculate that the patients in this study were chronic kidney disease caused by glomerular nephropathy, and the pathological changes are similar, so the changes in renal perfusion have less variability. Perfusion values and eGFR displayed no correlation in healthy volunteers in this study. The reason might be that renal blood flow in the healthy volunteers is autoregulatory, so GFR maintains constant across a wide range of renal blood flow. All of above indicated the breath-holding FAIR-ASL technique could be a non-invasive approach to assist the diagnosis of mild PGD and monitoring renal function impairment in $\mathrm{PGD}$ patients.

A few limitations of this study should be noted. First, we did not use the para-aminohippurate (PAH) clearance rate, the gold standard for renal function assessment, which might lead to some shortcomings in the assessment. However, this method has technical problems in daily use, and eGFR is more commonly used and it is noninvasive in clinical work (17). Second, one study revealed that T1 relaxation time was higher in CKD within the cortex and whole kidney (18). The applied T1 relaxation time in our study was similar to many studies (7), and the perfu- 

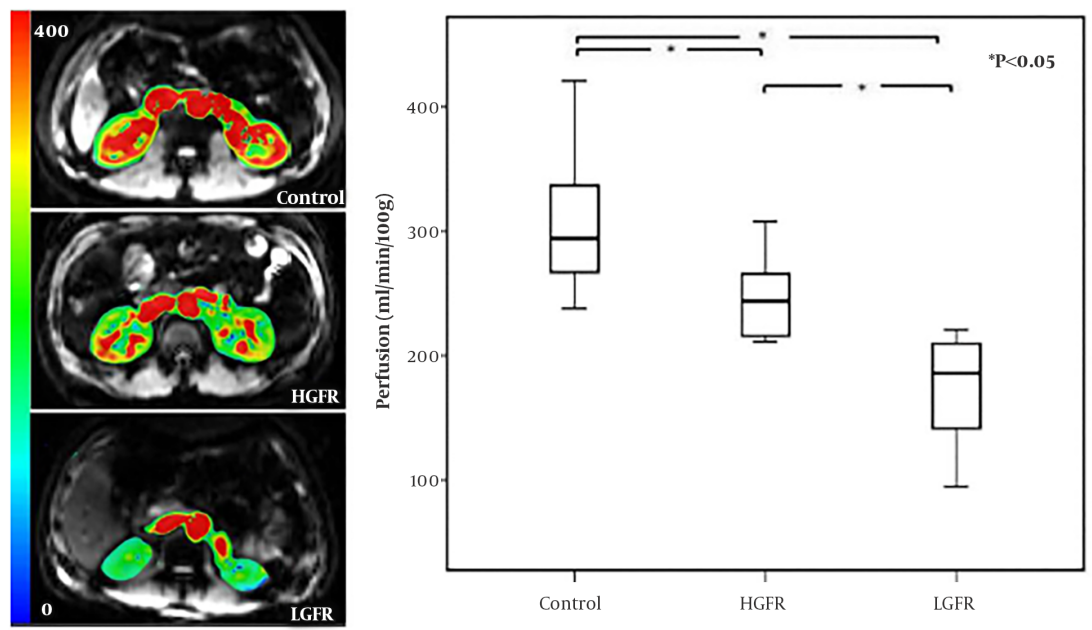

Figure 5. Comparison of perfusion among control, HGFR and LGFR group. The three images on the left are representative images of the kidney ASL in the control, HGFR and LGFR group. The right is the box plot of the comparison of perfusion values among the three groups. (HGFR, high estimated glomerular filtration rate; LGFR, low estimated glomerular filtration rate; ASL, arterial spin labeling)
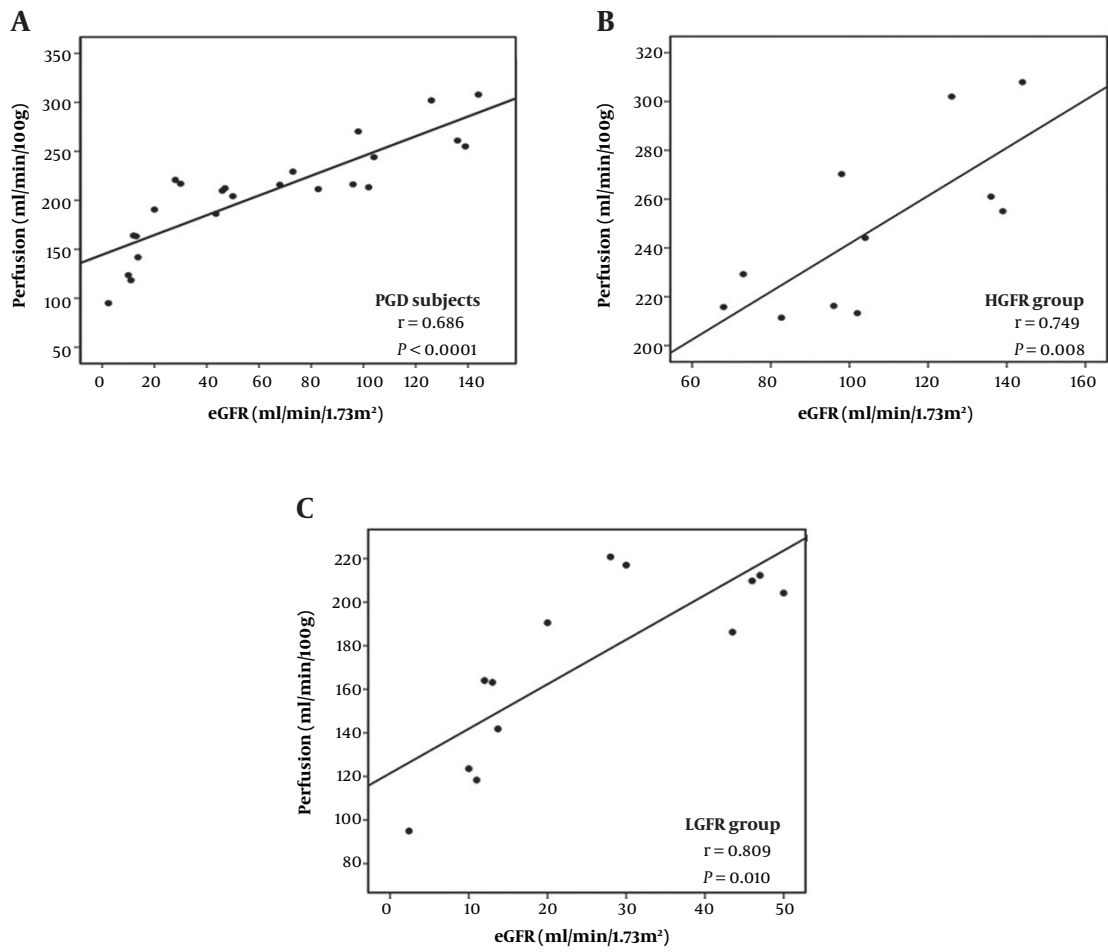

Figure 6. Spearman's correlation analysis of perfusion with eGFR in all PGD patients (A), high eGFR patients (B) and low eGFR patients (C).(PGD, primary glomerular disease; eGFR, estimated glomerular filtration rate)

sion values in healthy volunteers were similar to the study that used changed T1 relaxation time. Third, the patients in this study were divided into HGFR and LGFR groups due to the relatively small number of different CKD grades in
PGD patients. Thus, further analysis based on a large sample size is required to clarify the relationships between the different causes and stages of PGD.

In conclusion, the breath-holding FAIR-ASL technique 
performed a feasible and non-radioactive approach to monitor the renal perfusion variations corresponding to eGFR in PGD patients. It would be a noninvasive technique to discover the early hemodynamic changes of renal function and assist the diagnosis of mild PGD.

\section{Footnotes}

Authors' Contributions: Chenxia Li and Xiang Li contributed equally as the first author. Study concept and design: Yuelang Zhang and Chenxia Li; acquisition of data: Xiang Li and Haitian Liu; analysis and interpretation of data: Rong Wang and Gang Niu; drafting of the manuscript: Chenxia Li and Xiang Li; critical revision of the manuscript for important intellectual content: Yuelang Zhang and Jian Yang; statistical analysis: Chenxia Li and Xiang Li; administrative, technical, and material support: Yuelang Zhang and Jian Yang; study supervision: Yuelang Zhang

Conflict of Interests: The authors declare that they have no conflict of interest.

Ethical Approval: This case-control study was approved by the Ethics Committee of our hospital.

Funding/Support: This work was supported by the Shaanxi Provincial Key Research and Development Program [2019SF-007].

Informed Consent: All subjects signed an informed consent prior to the examination.

\section{References}

1. Jha V, Garcia-Garcia G, Iseki K, Li Z, Naicker S, Plattner B, et al. Chronic kidney disease: Global dimension and perspectives. Lancet. 2013;382(9888):260-72. doi: 10.1016/S0140-6736(13)60687-X. [PubMed: 23727169].

2. Zhang L, Wang F, Wang L, Wang W, Liu B, Liu J, et al. Prevalence of chronic kidney disease in China: A cross-sectional survey. Lancet. 2012;379(9818):815-22. doi: 10.1016/S0140-6736(12)60033-6. [PubMed: 22386035].

3. Li C, Liu H, Li X, Zhou L, Wang R, Zhang Y. Application of BOLDMRI in the classification of renal function in chronic kidney disease. Abdom Radiol (NY). 2019;44(2):604-11. doi: 10.1007/s00261-018-1750-6. [PubMed: 30151714].

4. Klessens CQ, Woutman TD, Veraar KA, Zandbergen M, Valk EJ, Rotmans JI, et al. An autopsy study suggests that diabetic nephropathy is underdiagnosed. Kidney Int. 2016;90(1):149-56. doi: 10.1016/j.kint.2016.01.023. [PubMed: 27165826].

5. Heusch P, Wittsack HJ, Blondin D, Ljimani A, Nguyen-Quang M, Martirosian $\mathrm{P}$, et al. Functional evaluation of transplanted kidneys using arterial spin labeling MRI. J Magn Reson Imaging. 2014;40(1):84-9. doi: 10.1002/jmri.24336. [PubMed: 24123319].
6. Niles DJ, Artz NS, Djamali A, Sadowski EA, Grist TM, Fain SB. Longitudinal assessment of renal perfusion and oxygenation in transplant donor-recipient pairs using arterial spin labeling and blood oxygen level-dependent magnetic resonance imaging. Invest Radiol. 2016;51(2):113-20. doi: 10.1097/RLI.0000000000000210. [PubMed: 26561047]. [PubMed Central: PMC4697870].

7. Li LP, Tan H, Thacker JM, Li W, Zhou Y, Kohn O, et al. Evaluation of renal blood flow in chronic kidney disease using arterial spin labeling perfusion magnetic resonance imaging. Kidney Int Rep. 2017;2(1):3643. doi: 10.1016/j.ekir.2016.09.003. [PubMed: 28868513]. [PubMed Central: PMC5575771].

8. Roberts DA, Detre JA, Bolinger L, Insko EK, Lenkinski RE, Pentecost MJ, et al. Renal perfusion in humans: MR imaging with spin tagging of arterial water. Radiology.1995;196(1):281-6. doi: 10.1148/radiology.196.1.7784582. [PubMed: 7784582].

9. Karger N, Biederer J, Lusse S, Grimm J, Steffens J, Heller M, et al. Quantitation of renal perfusion using arterial spin labeling with FAIRUFLARE. Magn Reson Imaging. 2000;18(6):641-7. doi: 10.1016/s0730725x(00)00155-7. [PubMed: 10930773].

10. Mora-Gutierrez JM, Garcia-Fernandez N, Slon Roblero MF, Paramo JA, Escalada FJ, Wang DJ, et al. Arterial spin labeling MRI is able to detect early hemodynamic changes in diabetic nephropathy. J Magn Reson Imaging. 2017;46(6):1810-7. doi: 10.1002/jmri.25717. [PubMed: 28383796].

11. Ma YC, Zuo L, Chen JH, Luo Q, Yu XQ, Li Y, et al. Modified glomerular filtration rate estimating equation for Chinese patients with chronic kidney disease. J Am Soc Nephrol. 2006;17(10):2937-44. doi: 10.1681/ASN.2006040368. [PubMed: 16988059].

12. Hammon M, Janka R, Siegl C, Seuss H, Grosso R, Martirosian P, et al. Reproducibility of kidney perfusion measurements with arterial spin labeling at 1.5 tesla MRI combined with semiautomatic segmentation for differential cortical and medullary assessment. Medicine (Baltimore). 2016;95(11). e3083. doi: 10.1097/MD.0000000000003083. [PubMed: 26986143]. [PubMed Central: PMC4839924].

13. Becker AS, Rossi C. Renal arterial spin labeling magnetic resonance imaging. Nephron. 2017;135(1):1-5. doi: 10.1159/000450797. [PubMed: 27760424].

14. Gillis KA, McComb C, Foster JE, Taylor AH, Patel RK, Morris ST, et al. Inter-study reproducibility of arterial spin labelling magnetic resonance imaging for measurement of renal perfusion in healthy volunteers at 3 Tesla. BMC Nephrol. 2014;15:23. doi: 10.1186/1471-2369-15-23. [PubMed: 24484613]. [PubMed Central: PMC3909760].

15. Musso CG, Oreopoulos DG. Aging and physiological changes of the kidneys including changes in glomerular filtration rate. Nephron Physiol. 2011;119 Suppl 1:p1-5. doi: 10.1159/000328010. [PubMed: 21832859].

16. Rossi C, Artunc F, Martirosian P, Schlemmer HP, Schick F, Boss A. Histogram analysis of renal arterial spin labeling perfusion data reveals differences between volunteers and patients with mild chronic kidney disease. Invest Radiol. 2012;47(8):490-6. doi: 10.1097/RLI.0b013e318257063a. [PubMed: 22766911].

17. Kalantarinia K, Belcik JT, Patrie JT, Wei K. Real-time measurement of renal blood flow in healthy subjects using contrast-enhanced ultrasound. Am J Physiol Renal Physiol. 2009;297(4):F1129-34. doi: 10.1152/ajprenal.00172.2009. [PubMed: 19625375]. [PubMed Central: PMC2775574].

18. Gillis KA, McComb C, Patel RK, Stevens KK, Schneider MP, Radjenovic $A$, et al. Non-contrast renal magnetic resonance imaging to assess perfusion and corticomedullary differentiation in health and chronic kidney disease. Nephron. 2016;133(3):183-92. doi: 10.1159/000447601. [PubMed: 27362585]. 